\title{
Mentalisierungsbasierte Gruppentherapie bei Erwachsenen mit Autismus-Spektrum-Störung
}

\author{
Sophia Reul, Ulrich Schultz-Venrath, Kai Vogeley, Katharina Krämer
}

\begin{abstract}
Autismus-Spektrum-Störungen sind gekennzeichnet durch Schwierigkeiten in der Interaktion und der Kommunikation sowie repetitive, stereotype Verhaltensweisen und Interessen. Hierbei werden besonders die Einschränkungen im Mentalisieren der Betroffenen als ursächlich für ihre interpersonalen Probleme diskutiert. Da das Mentalisieren bei der mentalisierungsbasierten Gruppentherapie explizit angeregt wird, erscheint sie auch für die Behandlung von ASS eine vielversprechende Methode zu sein. In einem Pilotprojekt wird sie nun erstmals in dieser Patientengruppe angewendet.
\end{abstract}

\section{Mentalisierungsbasierte Gruppentherapie (MBT-G)}

Die mentalisierungsbasierte Gruppentherapie (MBT-G) ist eine neuere Entwicklung psychodynamischer Gruppenpsychotherapien. Sie wurde von der Arbeitsgruppe um Antony Bateman und Peter Fonagy in einer Londoner Tagesklinik für die Behandlung von Menschen mit einer BorderlinePersönlichkeitsstörung (BPS) konzipiert [1]. Das Mentalisierungskonzept fußt auf der Bindungstheorie von Bowlby und auf der Affekttheorie von Panksepp und Biven. Bezüglich der kognitiven Aspekte finden sich Überschneidungen mit der Theory-of-Mind-Forschung (ToM).

\section{Merke}

Mentalisieren beschreibt die Fähigkeit, sich auf mentale Zustände (z. B. Überzeugungen, Gedanken, Gefühle, Bedürfnisse und Wünsche) von sich und anderen zu beziehen und diese als dem Verhalten zugrundeliegend zu begreifen und darüber nachdenken zu können [1].

Auf diese Weise kann ein Individuum sein inneres Erleben und das anderer Personen gleichermaßen wahrnehmen und in die eigene Handlungsplanung einbinden. Für zwischenmenschliche Begegnungen ist diese Form der Perspektivübernahme von zentraler Bedeutung und eine wichtige Voraussetzung, um die Interaktion mit anderen mühelos, automatisch und intuitiv gestalten zu können.

Fokus der MBT-G ist die Förderung des Mentalisierens durch gezielte explizite Interventionen. Aktuell existieren 3 Manuale zur Durchführung von MBT-G, die sich mehr als eine Art Leitfaden anstatt einer strengen Anleitung verstehen lassen. Für die Behandlung von BPS wird MBT-G seit Jahren sehr erfolgreich eingesetzt. Inzwischen wurde ihre Wirksamkeit auch für PatientInnen mit anderen psychischen Erkrankungen nachgewiesen (z. B. antisoziale, narzisstische Persönlichkeitsstörungen, Depressionen, Essstörungen) [1][2][3]. Menschen mit Autismus-Spektrum-Störungen wurden bisher noch nicht damit behandelt.

\section{Dimensionen des Mentalisierens und prämentalistische Modi}

Mentalisieren lässt sich mit 4 Dimensionen beschreiben ( Tab. 1) [1][2][3][4], die neurobiologisch begründet sind [14]. Schwierigkeiten des Mentalisierens treten dann auf, wenn zu stark auf einen Pol einer Dimension fokussiert wird, wodurch die Aspekte der anderen Dimension vernachlässigt werden. Gelingendes Mentalisieren entsteht hingegen dann, wenn beide Pole einer Dimension und schließlich alle Dimensionen gleichermaßen fluktuierend vom Individuum genutzt werden und ausgewogen präsentiert sind. Neben diesen Dimensionen kann entwicklungspsychologisch zwischen drei, bzw. vier prämentalistischen Modi unterschieden werden. Sie erfordern spezifische Interventionen zur Förderung des Mentalisierens. ( Tab. 2) $[4,5]$.

\section{Autismus-Spektrum-Störungen im Erwachsenenalter}

\section{Kernsymptome und Merkmale}

Autismus-Spektrum-Störungen (ASS) werden im DSM-5 und im ICD-10 als tiefgreifende Entwicklungsstörung klassifiziert, die durch die Kernsymptome Störungen der Interaktion und der Kommunikation sowie durch repetitive, stereotype Verhaltensweisen und Interessen gekennzeichnet sind [6][7]. Hierbei lassen sich besonders die Komplikationen in der sozialen Interaktion und in verbalen sowie 
- Tab. 1 Dimensionen des Mentalisierens (nach [2])

implizit: automatisch, schnell, reflexhaft, unbewusst

selbst:

Fokus auf eigene mentale

Zustände

kognitiv:

Gedanken und Schlussfolge-

rungen von sich und anderen

verstehen/reflektieren, Gefahr zum „Pseudomentalisieren“

internal:

innere Zustände, Gedanken,

Gefühle, Wünsche vs. explizit:

kontrolliert, langsam, bewusst

vs. andere:

Fokus auf mentale Zustände anderer

vs. affektiv:

Gefühle von sich und anderen verstehen/reflektieren, emotionale

Überempfindlichkeit

vs. external:

Mimik, Gestik, Stimme, Verhalten
>Tab. 2 Prämentalistische Modi (nach [4, 5])

\begin{tabular}{|l|l|}
\hline Modus & Beschreibung \\
\hline Körpermodus & $\begin{array}{l}\text { Prä- und postnatal (etwa bis zum 9. Monat) kann der } \\
\text { Säugling Körper und Psyche noch nicht getrennt erleben. } \\
\text { Er ist primär mit seinen Körper- und Hautempfindungen } \\
\text { beschäftigt. }\end{array}$ \\
\hline teleologischer & $\begin{array}{l}\text { Handlungen werden auf Basis ihrer physischen anstatt ihrer } \\
\text { mentalen Bedingungen verstanden. Nur real befriedigende } \\
\text { Modus }\end{array}$ \\
$\begin{array}{l}\text { Handlungen und physisch manifeste Veränderungen können } \\
\text { das eigene mentale Erleben beeinflussen. }\end{array}$ \\
\hline Äquivalenz- & $\begin{array}{l}\text { Die innere Welt und die äußere Realität werden als iden- } \\
\text { tisch wahrgenommen und erlebt. Es gibt kaum Differenzen } \\
\text { zwischen Fantasie und Realität und es besteht eine hohe } \\
\text { Intoleranz gegenüber alternativen Perspektiven sowie eine } \\
\text { konkretistische Wahrnehmung. }\end{array}$ \\
\hline Als-ob-Modus & $\begin{array}{l}\text { Die innere Welt ist von der externen Wirklichkeit entkoppelt, } \\
\text { d. h. der mentale Zustand hat keine Implikation für die } \\
\text { Außenwelt und umgekehrt. Dabei kommt es zu detaillierten, } \\
\text { aber oberflächlichen Beschreibungen und Darstellungen } \\
\text { vermeintlich mentaler Zustände, die in Wirklichkeit } \\
\text { bedeutungslos und nicht authentisch sind. Man spricht } \\
\text { in diesem Fall auch von „Pseudomentalisieren“ oder von } \\
\text { „Hypermentalisieren“. }\end{array}$ \\
\hline
\end{tabular}

nonverbalen Kommunikationsmustern mit Abweichungen in der ToM-Entwicklung bzw. im Mentalisieren in Verbindung bringen [8].

\section{ToM bei ASS}

Die Fähigkeit zur ToM entwickelt sich stufenweise im Laufe der ersten 5 Lebensjahre eines Kindes. Während Kinder ohne ASS bereits im 1. Lebensjahr die Fähigkeit zur ,joint attention “ zeigen und im Alter von 4-5 Jahren i. d. R. formalisierte ToM-Aufgaben lösen können, weisen Kinder mit ASS eine deutliche Entwicklungsverzögerung in diesem Bereich auf [8].

\begin{abstract}
Merke
Erwachsene mit ASS ohne Intelligenzminderung sind zumeist in der Lage, regelbasiert formalisierte Mentalisierungsaufgaben in Form von Testaufgaben zu lösen, haben aber in komplexen Alltagssituationen immense Probleme in der impliziten Erfassung mentaler Zustände anderer Menschen.
\end{abstract}

Meist sind diese auf differenzielle Störungen in der impliziten Verarbeitung sozial relevanter und besonders nonverbaler Informationen zurückzuführen. So werden nonverbale Kommunikationssignale (wie Mimik, Gestik und Blickverhalten) weder zu kommunikativen Zwecken eingesetzt noch als Quelle sozial relevanter Informationen herangezogen. In der verbalen Kommunikation kommt es vor allem zu Abweichungen der Sprache in ihrer sozial-kommunikativen Form, z. B. in Form von Monologisieren, Unterbrechen an unpassenden Stellen, fehlender Prosodie.

Wie beim Lösen von Mentalisierungsaufgaben erlernen Personen mit ASS ohne Intelligenzminderung häufig gewisse kommunikative Kompetenzen, sodass eindeutige nonverbale Signale (starke, deutliche Gesichtsausdrücke oder Gesten) bewusst analysiert und richtig interpretiert werden können, komplexere oder subtilere nonverbale Signale jedoch meist nicht oder falsch wahrgenommen werden. Generell erscheinen inhaltlich-verbale Informationen für Personen mit ASS im Umgang mit anderen Menschen verlässlicher und werden daher von ihnen meist stärker gewichtet. Darüber hinaus lassen sich Auffälligkeiten, wie ein eingeschränktes Verständnis von Metaphern, von Ironie oder Witz oder der häufig falsche Einsatz von Floskeln erklären [6][9].

Insgesamt führt das Mentalisierungsdefizit bei Menschen mit ASS zu häufigen Unsicherheiten und Missverständnissen im Erleben und Einschätzen sozialer Situationen und im wechselseitigen emotionalen Miteinander. Die Dauerbelastung durch „mentalen Stress“ und die damit einhergehende soziale Isolation kann bei den Betroffenen einen erheblichen Leidensdruck hervorrufen. Nicht selten kommt es zur Ausbildung komorbider psychischer Störungen wie Depressionen, Angsterkrankungen, Tic- oder Zwangsstörungen.

\section{Psychotherapie bei ASS im Erwachsenenalter}

Die psychotherapeutische Behandlung von erwachsenen Personen mit ASS hat sich als sehr hilfreich und sinnvoll erwiesen [10]. Allerdings bedarf es bei der Umsetzung einiger Besonderheiten. Das betrifft zunächst die PatientIn-Therapeutln-Beziehung: Aufgrund der Störung in der sozialen Informationsverarbeitung bei ASS wird von TherapeutInnen eine spezielle Sensibilisierung und ein adaptiertes Beziehungsangebot gefordert [11]. Des Weiteren ist zu beachten, dass ASS nicht ursächlich behandelt werden kann. Vielmehr sollen die Erweiterung des Verhaltens- 
repertoires, verbesserte Bewältigungsstrategien und die Behandlung komorbider Symptome unterstützt werden [10].

Obwohl gruppentherapeutische Konzepte für diese Patientlnnengruppe spezielle Herausforderungen mit sich bringen, bieten sie gleichzeitig auch ein besonderes Entwicklungspotenzial [10]: So sind multiple soziale Interaktionen, wie es eine Gruppe erfordert, für Menschen mit ASS aufgrund ihrer typischen Defizite und ihren häufigen negativen Erfahrungen zunächst meist angstbesetzt. In einem durch die TherapeutInnen strukturierten und geschützten Rahmen werden im Verlauf jedoch allgemeine gruppentherapeutische Wirkfaktoren (z.B. Gruppenkohäsion, Universalität des Leidens und Hoffnungsvermittlung) wirksam und führen zu neuen, positiven Beziehungserfahrungen [12]. Der identitätsstiftende Faktor einer Gruppe kann einen besonders nachhaltigen und heilsamen therapeutischen Einfluss haben [10]. Ein weiterer Vorteil gruppentherapeutischer Konzepte ist, dass die Gruppe einen idealen Übungsraum bietet, um das komplexe Netz sozialer Interaktion aus eigenen Bedürfnissen, Gefühlen und Handlungsintentionen und denen der anderen zu registrieren, zu analysieren und verstehen zu lernen.

\section{MBT-G bei ASS}

In der Spezialambulanz für Autismus im Erwachsenenalter der Klinik und Poliklinik für Psychiatrie und Psychotherapie der Uniklinik Köln wird MBT-G erstmalig in der Behandlung von Personen mit ASS im Erwachsenenalter angewendet und wissenschaftlich begleitet.

\section{Projektbeschreibung}

Ziel des Forschungsprojekts ist zu untersuchen, ob Erwachsene mit ASS von der Behandlung mit MBT-G profitieren, um im Falle eines positiven Resultats das psychotherapeutische Angebot für Menschen mit ASS nachhaltig erweitern zu können. Die Gesamtdauer des Projekts umfasst einen Zeitraum von 2 Jahren (2018-2020), in welchem 2 geschlossene Therapiegruppen mit jeweils 10 Teilnehmerlnnen und 2 Therapeutinnen einen Therapiezyklus von 20 MBT-G-Sitzungen ( 1 × wöchentlich) durchlaufen. Die Wirksamkeit von MBT-G wird dabei sowohl durch quantitative als auch durch qualitative Erhebungsmethoden überprüft. Zur Adhärenzprüfung werden die Sitzungen mit Hilfe von Video- und Tonbandaufnahmen dokumentiert. Vor Beginn des Projekts wurde zunächst eine „Feasibility“-Studie (9 TeilnehmerInnen, 12 Sitzungen) durchgeführt.

\section{Beeinträchtigungen des Mentalisierens bei ASS}

Die Mentalisierungsfähigkeit von Personen mit ASS lässt sich anhand der Dimensionen des Mentalisierungsmodells folgendermaßen beschreiben:
Implizit vs. explizit

Da Personen mit ASS große Schwierigkeiten haben, spontan zu mentalisieren [8][9], wodurch ihnen in sozialen Situationen häufig die Orientierung fehlt, verwenden sie verstärkt einen expliziten Modus des Mentalisierens. Dieser Modus hilft ihnen, die Situation bewusst zu analysieren, erlernte soziale Reaktionen und Verhaltensweisen auf ihre mögliche Passung hin zu prüfen und die jeweilige Interaktion besser zu verstehen und damit auch besser kontrollieren zu können.

Selbst vs. andere

Der Aufmerksamkeitsfokus liegt bei Personen mit ASS primär auf den eigenen mentalen Zuständen, die im Allgemeinen auch als unabhängig von anderen wahrgenommen werden. Das „Hineinversetzten“ in andere findet i. d. R. erst dann statt, wenn das Verhalten des anderen zu Irritation führt und die Aufmerksamkeit willentlich darauf gelenkt wird.

\section{Kognitiv vs. affektiv}

Für diese Dimension lässt sich kein eindeutiger Fokus für Personen mit ASS bestimmen. Vielmehr muss zwischen dem Umgang mit eigenen mentalen Zuständen und denen anderer unterschieden werden. Bezogen auf die eigenen mentalen Zustände ist das Mentalisieren bei ASS eher dem affektiven Pol zuzuordnen. Das bedeutet, dass sie oftmals Probleme haben, ihre Emotionen zu regulieren, zu dosieren und diese mit situativen Elementen in Verbindung zu bringen. In Bezug auf die mentalen Zustände anderer ist ihr Mentalisieren stärker kognitiv ausgerichtet.

In Interaktionen mit anderen nehmen Individuen mit ASS Gefühle häufig erst dann wahr, wenn das Gegenüber diese deutlich vermittelt oder erklärt. Dadurch ist bei der Verarbeitung mentaler Zustände anderer vermutlich fast immer ein kognitiver Prozess vorgeschaltet, bevor es zu einer affektiven Anteilnahme kommen kann. Dies unterscheidet sie von Personen mit einer BPS, die sich häufig weder von den eigenen, noch von den Affektzuständen ihrer Umwelt distanzieren können und förmlich davon „überrollt“ werden [3].

\section{Internal vs. external}

ASS-Individuen haben sowohl mit dem Mentalisieren innerer als auch äußerer Aspekte Probleme. Aufgrund ihrer eingeschränkten Fähigkeit zur spontanen Perspektivübernahme und zur kognitiven Empathie [13] fällt es ihnen schwer, den Fokus auf innere mentale Prozesse zu richten. Gleichzeitig haben sie auch Schwierigkeiten in der Verarbeitung nonverbaler Kommunikationssignale und können äußere Hinweisreize mentaler Zustände nur eingeschränkt wahrnehmen [6][9]. Allgemein zeigen Erwachsene mit ASS eher eine Tendenz zum externalen Mentalisieren, indem sie sich in sozialen Situationen an offensichtlichen äußeren Hinweisreizen mentaler Zustände wie Mimik und Gestik orientieren und versu- 
chen, deren Bedeutung aktiv zu erlernen. Allerdings ist das Erlernte nicht ohne weiteres auf komplexe soziale Situationen übertragbar, weshalb es häufig zu Fehldeutungen oder Überinterpretationen, z. B. von Gesichtsausdrücken, kommt [6].

\section{MBT-G-Interventionen bei ASS}

Im Folgenden werden einzelne spezifische Aspekte der MBT-G-Behandlung von ASS auf Grundlage von den bisherigen klinischen Erfahrungen und Beobachtungen dargestellt.

\section{Rahmenbedingungen und Regulieren von Gruppenphasen}

Die MBT-G ist im Gegensatz zu den bisher beschriebenen verhaltenstherapeutischen Manualen für ASS vergleichsweise wenig strukturiert. Der „offene“ Gesprächsraum, in welchem weder die Themen vorgegeben noch die Reihenfolge oder der Anteil der Sprecher geregelt ist, kann zunächst Unsicherheit und Unbehagen bei den TeilnehmerInnen auslösen. Es sollte daher in der Anfangsphase überdurchschnittlich viel Zeit darauf verwendet werden, mit der Gruppe individuell sinnvolle Rahmenbedingungen zu erarbeiten und detailliert in die Methode und deren Ziele einzuführen. In dem vorliegenden Projekt wurden zu diesem Zweck 4 Einführungssitzungen mit den Themen Mentalisieren, Psychoedukation ASS, Emotionen und MBT-G durchgeführt.

\section{Nicht wissende Haltung}

Die „nicht wissende Haltung“ ist zentral im Mentalisierungsmodell und sieht vor, dass die TherapeutInnen aus einer (möglichst) natürlichen Neugier heraus explorieren, indem sie nachfragen und dabei dem Prozess der Gruppe eher folgen statt ihn zu leiten [1][4].

Dies kann als eine Art Weiterführung der allgemeinen Besonderheit in der therapeutischen Arbeit mit ASS angesehen werden, in der Therapeut Innen sich generell nicht auf die eigene Interpretation nonverbaler Signale von PatientenInnen verlassen, sondern vielmehr gezielt nachfragen sollten [10]. Darüber hinaus hat das gemeinsame Gruppenziel, interaktionelle Prozesse verstehen und mentalisieren zu wollen, für die PatientInnen eine Modellfunktion, indem ihnen wiederholtes und gezieltes Nachfragen einen Weg aufzeigt, um mit dem anderen in Kontakt und zu einem authentischen Austausch zu kommen.

Die nicht wissende Haltung erweist sich jedoch noch aus anderen Gründen als zentral in der Behandlung von ASS: Menschen mit ASS fühlen sich aufgrund negativer Erfahrungen in Gruppensituationen häufig abgelehnt, isoliert und missverstanden. Viele berichten, solche Gefühle explizit im Kontakt mit TherapeutInnen oder BehandlerInnen erlebt zu haben. Die in der MBT-G geforderte vorurteilsfreie, respektvoll und authentische Haltung der TherapeutInnen soll für die GruppenteilnehmerInnen eine vertrau- ensvolle Atmosphäre schaffen. Gelingt dies, kann das im weiteren therapeutischen Verlauf sogar als korrigierende Beziehungserfahrung gewertet werden.

\section{Emotionen fokussieren}

Das gemeinsame Explorieren, Reflektieren und Klären von Emotionen ist ein Kernaspekt der MBT-G [1][4]. In der Arbeit mit ASS sind dabei folgende Dinge zu beachten:

Aufgrund des erhöhten Selbst-Fokus der PatientInnen ist es wichtig, aktiv die Reflexion anderer Perspektiven anzuleiten („contrary moves“) und damit ein vollständigeres Bild einer zwischenmenschlichen Interaktion zu erreichen. Emotionen können von den TeilnehmerInnen häufig differenziert benannt werden, jedoch fehlt ihnen meist eine Idee zu deren Entstehung. Ist der kognitive Pol des Mentalisierens unterrepräsentiert, sollten TherapeutInnen weiter explorieren und nachfragen, bis ein umfassendes Bild des Gefühlszustands entsteht, das sowohl affektive als auch kognitive Elemente in der Reflexion vereint. Dabei ist darauf zu achten, dass sich die TherapeutInnen nicht zu sehr von ihren eigenen Ideen leiten lassen oder zu schnell überzeugt sind (d. h. eine nicht wissende Haltung bewahren). Die Gefahr ist sonst groß, in den Als-ob-Modus zu geraten, d. h. man gleitet in einen theoretischen Diskurs über innere Zustände, ohne die Situation mental wirklich zu erfassen.

Wichtig ist ebenfalls das Markieren von Emotionen durch die TherapeutInnen, indem immer wieder die im Gruppenprozess auftauchenden Emotionen aufgegriffen und gezielt benannt werden. Das Äußern eigener Gefühle und Gedanken im Sinne der Selbstöffnung des Therapeuten ist in der Arbeit mit ASS von besonderer Bedeutung, da er als „Experte“ für eine nicht autistische Wahrnehmung fungiert und den TeilnehmerInnen als Modell für nicht autistisches Verhalten zur Verfügung steht. Auch nonverbale Signale der Zustimmung oder der empathischen Anteilnahme sollten zusätzlich durch verbale Rückmeldung deutlich gemacht werden [10].

\section{TeilnehmerInnen in das Mentalisieren externer Ereignisse einbinden}

Das thematisch offene Setting lädt alle Gruppenmitglieder dazu ein, relevante Erlebnisse und Erfahrungen mit der Gruppe zu teilen. Die TherapeutInnen haben die Aufgabe, verschiedene Narrative zugunsten der gemeinsamen Exploration miteinander zu verbinden. Menschen mit ASS fällt es aufgrund ihrer ToM-Defizite besonders schwer, zwischen kontextrelevanten und unwichtigen Informationen in ihren Mitteilungen zu unterscheiden. Deshalb sollten Therapeutlnnen während der Berichterstattungen der TeilnehmerInnen besonders häufig nachfragen, ohne falsche Scham darüber, dass man den/die PatientIn vermeintlich nicht versteht. Vielmehr verdeutlicht das den Wunsch, dass man ihn/sie verstehen will und dass auch Nichtautisten Probleme mit dem Mentalisie- 
ren haben können, was wiederum Modellfunktion haben kann. Darüber hinaus bekommt die berichtende Person eine Rückmeldung, welche Informationen für ihr Gegenüber tatsächlich notwendig sind, um sie (kognitiv und affektiv) verstehen zu können.

\section{Mentalisieren von Ereignissen innerhalb der Gruppe}

Ebenso wie relevante externe Ereignisse von der Gruppe gemeinsam exploriert werden sollen, sollten auch alle relevanten Ereignisse, die innerhalb der Gruppensitzungen stattfinden, identifiziert und exploriert werden. Aufgrund des verstärkten Selbstfokus im Mentalisieren bei ASS braucht es dafür eine aktive Anleitung durch die TherapeutInnen, indem sie einerseits sensibel äußere Hinweisreize der Teilnehmerlnnen aufnehmen und ansprechen („markieren“) sowie andererseits ihre eigenen Wahrnehmungen und Empfindungen in Bezug auf die Gruppenteilnehmerlnnen formulieren.

\section{Mentalisieren der Beziehung}

Mentalisieren der Beziehung innerhalb der Gruppe beinhaltet sowohl die Beziehungen der Gruppenmitglieder untereinander als auch zu den TherapeutInnen. Diese besondere Form des Mentalisierens wird im Rahmen der Behandlung von PatientInnen mit ASS möglich, sobald eine vertrauensvolle Atmosphäre innerhalb der Gruppe entstanden ist. Auch hier ist wieder eine erhöhte Aktivität durch die TherapeutInnen gefragt, da es bei Erwachsenen mit ASS selten zu einer spontanen affektbetonten Reaktion kommt.

\section{FAZIT}

Die bisherigen klinischen Erfahrungen weisen darauf hin, dass sich Personen mit ASS ohne Intelligenzminderung gut auf die Behandlung mit MBT-G einlassen können, wenn BehandlerInnen die Methode transparent und zielgruppengerecht einführen und gezielt die Gruppenbildung unterstützen. Wie Personen mit ASS MBT-G in einer heterogenen, nicht autismusspezifischen Therapiegruppe annehmen würden, lässt sich bisher nicht beurteilen. Ausgehend von Patientenberichten scheinen sie vor allem dadurch zu profitieren, dass sie Gelerntes in ihrer alltäglichen Beziehungsgestaltung fokussiert anwenden können, z. B. ihren Interaktionspartner konkret nach dessen Empfindungen oder Wahrnehmungen fragen.
Interessenkonflikt

Sophia Reul, Ulrich Schultz-Venrath, Kai Vogeley und Katharina Krämer erklären, dass sie sich bei der Erstellung des Beitrags von keinen wirtschaftlichen Interessen haben leiten lassen und im Zusammenhang mit diesem Beitrag keine Interessenkonflikte bestehen. Kai Vogeley hat Förderungen durch EU, DFG, ESF und LVR, vorwiegend im Bereich Autismus, sowie Tantiemen aus Buchverkäufen (Beltz-Verlag) erhalten. Das Projekt ASS und MBT-G wird von der Kämpgen-Stiftung (Köln) und der Marga und Walter Boll-Stiftung (Kerpen Sindorf) gefördert.

\section{Autorinnen/Autoren}

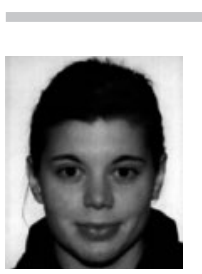

\section{Sophia Reul}

Dipl.-Psych., Psychologin und Psychotherapeutin (i. A.; TP, PA). Sie ist in der Klinik für Psychiatrie, Psychotherapie und Psychosomatik im EVK, Bergisch Gladbach, klinisch tätig. Darüber hinaus arbeitet sie als wissenschaftliche Mitarbeiterin in der Spezialambulanz für Autismus im Erwachsenenalter der Klinik und Poliklinik für Psychiatrie und Psychotherapie der Uniklinik Köln. Dort beschäftigt sie sich mit der Anwendung und Evaluation psychotherapeutischer Verfahren bei Autismus-SpektrumStörungen.

\section{Prof. Dr. med. Ulrich Schultz-Venrath} Privatpraxis für Psychosomatische Medizin und Psychotherapie, Gruppenanalyse und mentalisierungsbasierter Gruppentherapie. Ehemaliger Chefarzt der Klinik für Psychiatrie, Psychotherapie und Psychosomatik im EVK Bergisch Gladbach

Prof. Dr. Dr. Kai Vogeley

Klinik und Poliklinik für Psychiatrie und Psychotherapie, Uniklinik Köln

\section{Prof. Dr. Katharina Krämer}

Rheinische Fachhochschule Köln, Klinik und Poliklinik für Psychiatrie und Psychotherapie, Uniklinik Köln

\section{Korrespondenzadresse}

\section{Dipl.-Psych. Sophia Reul}

Klinik und Poliklinik für Psychiatrie und Psychotherapie Uniklinik Köln

Kerpener Str. 62

50937 Köln

sophia.reul@uk-koeln.de

\section{Literaturverzeichnis}

[1] Bateman A, Fonagy P, Hrsg. Handbook of Mentalizing in Mental Health Practice. 2. Aufl. Washington DC: American Psychiatric Publishing; 2019

[2] Bateman A, Fonagy P. Mentalization-Based Treatment for Personality Disorders: A Practical Guide. Oxford: OUP; 2016 
[3] Euler S, Schultz-Venrath U. Theorie und Praxis der mentalisierungs-basierten Therapie (MBT) bei der Borderline-Persönlichkeitsstörung. PiD 2014; 15(3): 40-43

[4] Schultz-Venrath U. Mentalisieren des Körpers. Stuttgart: Klett-Cotta; 2020

[5] Schultz-Venrath U, Felsberger H. Mentalisieren in Gruppen: Mentalisieren in Klinik und Praxis. Stuttgart: Klett-Cotta; 2016

[6] Vogeley K. Anders sein - Hochfunktionaler Autismus im Erwachsenenalter. 2. Aufl. Weinheim: Beltz; 2016

[7] DSM-5 Diagnostic Classification. In: Diagnostic and Statistical Manual of Mental Disorders. Im Internet: https://dsm.psychiatryonline.org/doi/abs/10.1176/appi. books.9780890425596.x00DiagnosticClassification; Stand: 23.05.2020

[8] Baron-Cohen S. Mindblindness. An Essay on Autism and Theory of Mind. Cambridge, MA: MIT Press; 1995

[9] Attwood T. The complete guide to Asperger's syndrome. London: Jessica Kinglsey Publishers; 2008

[10] Falter-Wagner CM, Vogeley K, Krämer K. Die besten Behandlungserfolge werden mit Psychotherapie erzielt. InFo Neurol Psych 2019; 21: 44-55
[11] Krämer K, Gawronski A, Falter-Wagner C et al. Die „doppelte Unsichtbarkeit" autistischer Störungen und ihre Herausforderungen für Psychotherapeuten und Angehörige. Psychother ] 2015; 3: 231-239

[12] Yalom ID. Theorie und Praxis der Gruppenpsychotherapie: ein Lehrbuch. Stuttgart: Klett-Cotta; 2007

[13] Dziobek I, Rogers K, Fleck S et al. Dissociation of Cognitive and Emotional Empathy in Adults with Asperger Syndrome Using the Multifaceted Empathy Test (MET). J Autism Dev Disord 2008; 38: 464-473

[14] Luyten P, Campbell C, Allison C et al. The Mentalizing Approach to Psychopathology: State of the Art and Future Directions. Ann Rev Clin Psychology 2020; 16: 297-325

Bibliografie

DOI https://doi.org/10.1055/a-0987-5642

PiD - Psychotherapie im Dialog 2020; 21: 71-76 (c) Georg Thieme Verlag KG Stuttgart · New York ISSN 1438-7026 